\title{
An n-body displacement framework for marine hydrodynamics
}

\author{
J. M. Niedzwecki, J. M. Roesset \& C. Xie \\ Zachry Department of Civil Engineering, \\ Texas A\&M University, College Station, TX, USA
}

\begin{abstract}
The wide variability of ocean platform designs, the increasingly common specification of multi-body configurations, and the random nature of the offshore environment present some interesting challenges. Experience with models of a single platform or a ship is well established and has provided an important linkage for the engineer to evaluate a design concept and provide a basis for design modifications prior to construction and installation offshore. The use of multiple models, i.e. n-bodies, in model tests is not new, but the procedures used in the analysis of the data are still evolving. This study presents a framework to study the data from n-body measurements and encompasses a wide range of offshore environments used in design. In order to illustrate the methodology experimental data for a two-body problem involving the coupled motion behaviour of a mini-TLP and tender barge subject to random waves is investigated. The selection of reference and relative scaling values are illustrated and discussed.
\end{abstract}

Keywords: $n$-body hydrodynamics, coupled response behaviour model tests, TLP model tests, random data analysis.

\section{Introduction}

The response behaviour of multi-body systems with varied degrees of coupling between the bodies or their closeness in proximity presents many challenges for the designers of offshore structures. Whether it is model tests, field installation or work-over operations in benign or more extreme design seas the relationships between the motions of the various bodies in the system require the development of a systematic approach to their interpretation. In order to better understand 
measured data as well as numerical simulations a consistent method for scaling that would lend itself to the verification of anticipated response behaviour would prove advantageous. Questions often arise as to the relative spacing, orientation and response behaviour of each body and the amplification of the fluid between each body. Froude scaling is the basis for most model tests in naval architecture and ocean engineering and it is there that is a logical starting point to develop a broader interpretation of multi-body systems. Further, the focus here is on deepwater floating bodies such as TLPs, Spars and barges that may utilize mooring systems, fendering systems dynamic positioning or some combination.

\section{Mathematical development}

\subsection{Froude scaling for the n-body problem}

For model basin test programs intended to study the response behaviour of offshore platforms or surface vessels the experiments are developed using Froude scaling (see for example Newman [1] or Chakrabarti [2]). There are a variety of forms that can be used to scale the geometric, kinematic and dynamic variables of interest. For this study a notation consistent with WAMIT [3] notation was selected and consequently the dimensionless forces and moments, $\bar{X}_{i}$ can be compactly expressed as

$$
\bar{X}_{i}=\frac{X_{i}}{\rho g A L^{m}}=\frac{X_{i}}{\rho g L^{m+1}}
$$

When the index, $m=2$, in equation (1) refers to the hydrodynamic forces of surge, sway and heave for the corresponding index, $i=1,2,3$. Similarly when the index, $m=3$, the corresponding hydrodynamic moments of roll, yaw and pitch are indicated for $i=4,5,6$ respectively. The remaining variables in equation (1) include $X_{i}$ the corresponding dimensional force or moment, $\rho$ the mass density of the fluid, $g$ the gravitational acceleration, $A$ the characteristic wave amplitude, and a characteristic length scale, $L$ selected for convenience.

For multi-body hydrodynamic problems there is no standard approach to developing a consistent dimensionless form for the various quantities of interest and one approach is to explore the use of vessel displacement related to the multi-body system. Consider the relative displacement, $\Delta$, defined as

$$
\Delta_{b: r}=\frac{\delta_{b}}{\delta_{r}}
$$

where $\delta$ is the individual body displacement of the n-bodies associated with the index $b=1,2, \ldots, n$ and the subscript $r$ indicates the reference displacement selected. This could be introduced directly, but experience suggests that a better approach would be to introduce a more general scaling, $s=C \Delta_{b: r}$ where $C$ is selected as an appropriate power of ten. The values of this constant will be explored in more detail in the examples to follow. Introducing these ideas into the equation (1) one obtains 


$$
\bar{X}_{b: i}=\frac{X_{b: i}}{s \rho g L_{r}^{m+1}}=\frac{X_{b: i}}{s g \delta_{r} L_{r}^{m-2}}
$$

\subsection{Displacement and rotations}

The development of the dimensionless ratios for displacements, relative displacements, rotations, and relative rotations follow the usual Froude scaling procedures utilizing a reference length or displacement scale and an angular reference scale selected as appropriate such that

$$
\bar{x}_{r: i}=\frac{x_{i}}{L_{r}}, \quad \Delta \bar{x}_{r: i}=\frac{x_{r}-x_{i}}{L_{r}}, \quad \bar{\theta}_{r: i}=\frac{\theta_{i}}{\theta_{r}} \quad \Delta \bar{\theta}_{r: i}=\frac{\theta_{r}-\theta_{i}}{\theta_{r}}
$$

where the order of displacements corresponds to $m=2$ and the order of the rotations corresponds to $m=3$.

\subsection{Spectral scaling for $n$-bodies}

The conversion of information for regular waves and time series of the data from laboratory for field experiments for n-body problems was discussed in the subsection 2.1. However, in some cases it maybe desirable to compute the various spectra in their dimensional form and then post-process the results to an appropriate dimensionless form. A complete set of single sided-spectral density equations, including cross-spectral density functions has been developed, but attention in this article focuses on the single sided cross-spectral displacement spectra, coherence and cross-correlation for the TLP barge system in their coupled and un-coupled configurations. Thus the appropriate equations are as follows

$$
\begin{aligned}
& \bar{G}_{x_{b i, i} x_{c j}}\left(f T_{r}\right)=\left(\frac{1}{s L_{r}^{2} T_{r}}\right) G_{x_{b i,} x_{c j}}(f) \\
& \bar{G}_{\theta_{b i,} \theta_{c j}}\left(f T_{r}\right)=\left(\frac{1}{s \theta_{r}^{2} T_{r}}\right) G_{\theta_{b i, i} \theta_{c, j}}(f)
\end{aligned}
$$

where in general the indices referring to the specific floating bodies are $b=1,2, \ldots, n$ and $c=1,2, \ldots, n$, and when $b=c$, the respective auto-spectrum are recovered otherwise the spectrum relate the measurements from different bodies. The range of the indices $\mathrm{i}$ and $\mathrm{j}$ must follow the earlier relation to the forces and moments but need not be identical. A reference time scale, $T_{r}$, associated with the response motions is introduced and allows one flexibility to verify the identity of various peaks in the spectrum and subsequently to visually evaluate their relative significance. Other frequency selections such as $\omega=2 \pi f, G(\omega)=G(f) / 2 \pi, \Omega=f T_{r}=f / f_{r}=\omega / \omega_{r}$, or $k r=\left(\omega^{2} / g\right) r \quad$ are easily accommodated. 


\section{Model test data and analysis}

The particulars of the models used in the test program can be found in Xie et al. [4]. A schematic of the experimental set-up is shown in Figure 1, where the head and beam sea orientations of the models are presented. Figure 2 shows an under water photograph of the actual models and the proximity of the models during the model tests, for a model scale of 1:62. The photograph clearly shows the TLP tendons, risers and the aft barge mooring line connectors.

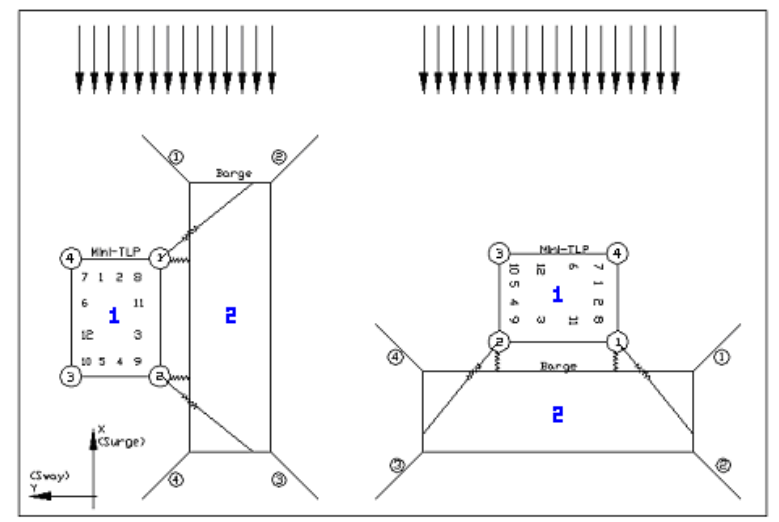

Figure 1: $\quad$ Model test set-up with respect to the incident design seas.

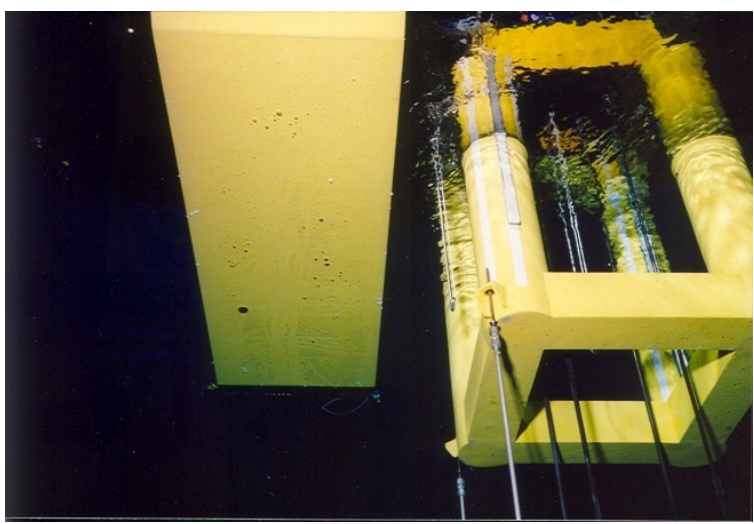

Figure 2: A photograph taken by a diver beneath the models.

The mini-TLP displacement was $10,318 \mathrm{t}$, the draft was $28.50 \mathrm{~m}$, and the column diameter was $8.75 \mathrm{~m}$ with a column separation of $28.5 \mathrm{~m}$. The pontoon had height of $6.25 \mathrm{~m}$ a width of $6.25 \mathrm{~m}$. The deck clearance was $10 \mathrm{~m}$. The original number of tethers was 8 , modelled as 4 and the original number of risers was 12 and modelled as 4 . The prototype mini-TLP had the following natural frequencies: surge $133 \mathrm{~s}$, sway $133 \mathrm{~s}$, heave $2.6 \mathrm{~s}$, yaw $121 \mathrm{~s}$ and pitch $4.9 \mathrm{~s}$. The 
tender barge had a length of $91.5 \mathrm{~m}$, a beam of $27.5 \mathrm{~m}$, and an operating draft of $3.7 \mathrm{~m}$. Some of the natural periods for the tender barge were: $107 \mathrm{~s}$ in surge, $125 \mathrm{~s}$ in sway and $48 \mathrm{~s}$ in yaw. The gap distance was $10 \mathrm{~m}$ and the relative displacement of the floating bodies was .827 .

\section{Data analysis}

The relative surge motion between the mini-TLP and tender barge is presented in Figure $3 \mathrm{a}$ and shows a large reduction in the mean as well as the fluctuations about the mean when the bodies are coupled. The motions normalized by the mini-TLP surge motion response are presented in Figure 3b, which illustrates that the coupled relative motion is about $20 \%$ of the uncoupled relative motion when the bodies are uncoupled.

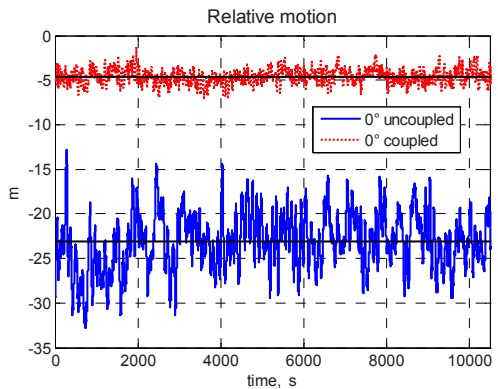

(a)

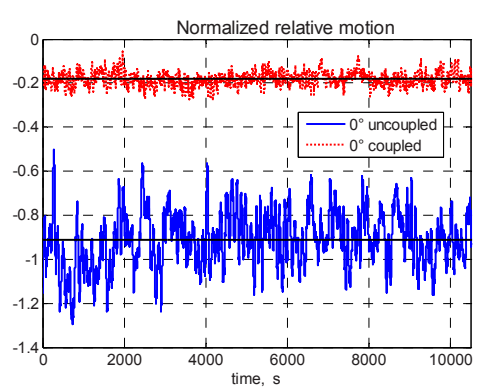

(b)

Figure 3: Relative surge motion for the uncoupled and coupled body configurations in head seas.

The roll and yaw motions of the tender barge when coupled to the mini-TLP normalized by the maximum tender barge pitch motion of 4.2 degrees is presented in Figure $4 \mathrm{a}$ and $4 \mathrm{~b}$ respectively. The maximum normalize roll is seen to be about a third of the maximum pitch and the yaw motion is seen to often exceed the maximum pitch motion.

The cross spectrum response, linear coherence and cross-correlation of the mini-TLP and tender barge surge motions are presented in Figure 5. The reference period selected is the natural period of the model mini-TLP in surge, 128s. That period corresponds to the first peak and the second peak at a dimensionless value of 8 corresponds to the peak period of the wave spectrum, 16s. As expected the linear correspondence around both peaks is quite high for the coupled configuration in head seas while for the uncoupled case where the bodies are independently moored the linear coherence indicates a much lower value as the mooring systems of the two bodies are quite different. More specifically, the mini-TLP tendons are highly pre-tensioned as compared to the catenary spread mooring configuration of the tender barge. The cross-correlation is presented in the usual fashion as a function of the lag. The oscillating peaks 
occur at spacing that is a multiple of the peak wave period, 16s. The difference between the coupled and uncoupled cases is strikingly different for the range of lags shown.

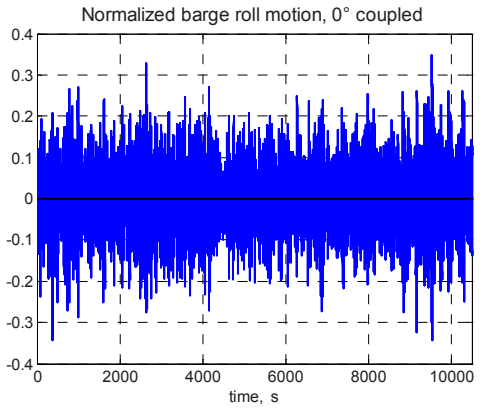

(a)

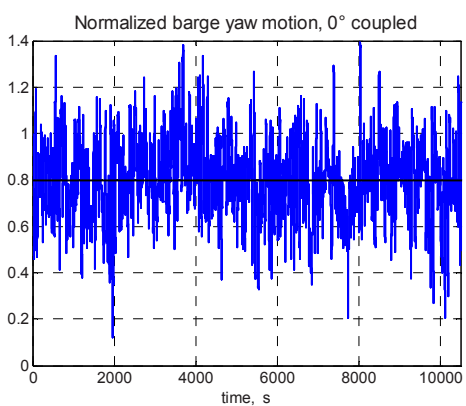

(b)

Figure 4: Tender barge roll and yaw motions normalized with maximum pitch for the coupled body configuration in head seas.
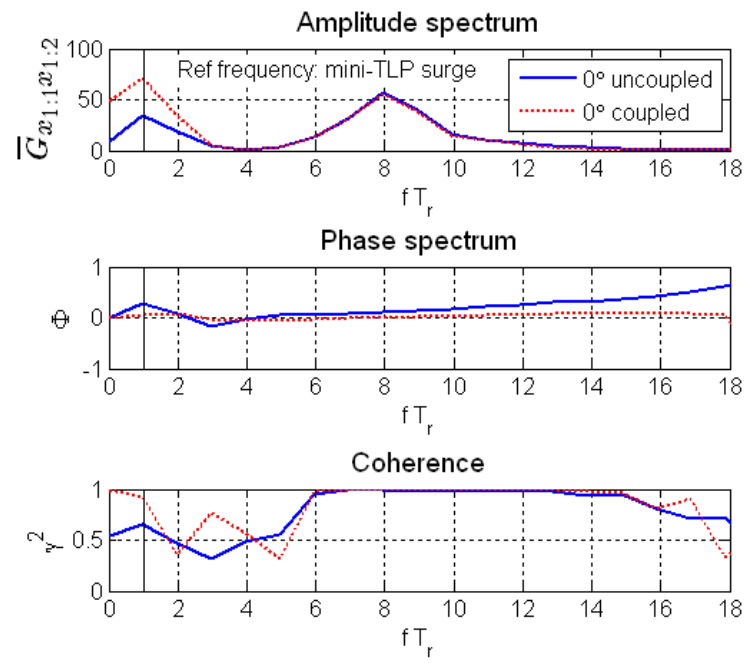

Cross correlation

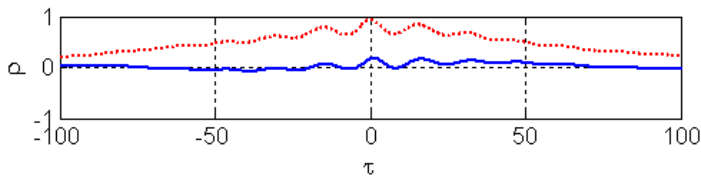

Figure 5: Characterization of the mini-TLP surge to the barge surge in head seas with reference to the mini-TLP natural period in surge, $128 \mathrm{~s}$. 
A comparison of the same data but with the peak period of the incident wave spectrum is presented in Figure 6. The basic interpretation of the cross-spectrum peaks is confirmed and illustrates how one could explore multi-peaked spectrum to verify the response behaviour at various natural platform response periods, environmental frequencies or for example sum and difference frequencies.

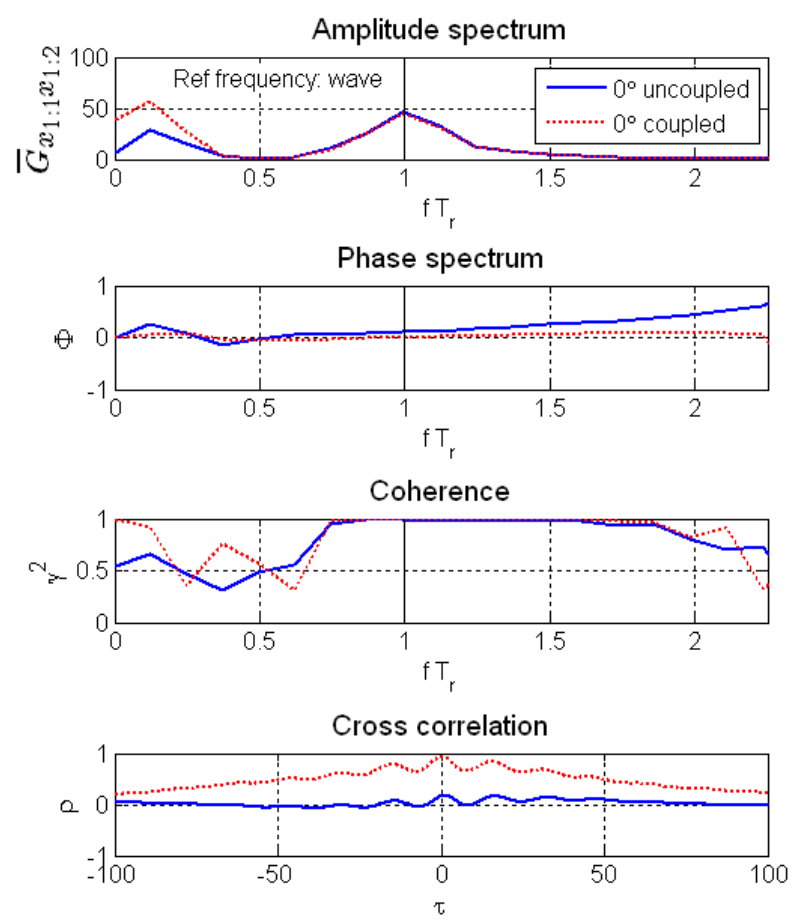

Figure 6: Characterization of the mini-TLP surge to the barge surge in head seas with reference to the peak wave spectrum period, $16 \mathrm{~s}$.

\section{Concluding remarks}

An approach to addressing the scaling and presentation of the dynamic response behaviour of n-body hydrodynamic was presented. The use of vessel displacement was introduced into Froude scaling using a scaling factor that appears as a constant in the dominator and later explicitly in Froude scaling. A notation was selected that would be consistent with WAMIT notation as that software has become a general standard in the offshore industry. In model testing the data are normally presented in prototype scale, which is consistent with the examples presented. The methodology presented was applied to relative motion behaviour as well as to cross-spectral analysis of the mini-TLP and tender barge response. The relative motions are seen to more easily compare the 
means and deviations of the data, while by selecting different reference frequencies it is relatively easy to verify the peaks at known natural periods or frequencies.

\section{References}

[1] Newman, J.N. (1977). Marine Hydrodynamics, MIT Press, Cambridge MA.

[2] Chakrabarti, S.K. (1994). Offshore Structure Modeling, World Scientific Publishing Company, River Edge, NJ.

[3] Newman, J.N. and Lee, C-H. (2008). WAMIT Users Guide, Chestnut Hill, MA.

[4] Xie, C., Niedzwecki, J.M. and Teigen, P.S. (2008). "Coupled Mini-TLP Barge Response in Random Seas," International Journal of Offshore and Polar Engineers, Vol. 18, No. 2, 1-8. 